\title{
SCREENING FOR ORGANIC ACID DISORDERS
}

\author{
Eliška Marklová, Miluše Brátová* and Viktor Vořǐšek** \\ Department of Pediatrics, Faculty Hospital, Charles University, Hradec Králové; \\ (Head: doc. MUDr. E. Pařízková, CSc.) \\ *Department of Gerontology \& Metabolism, Faculty Hospital, Charles University, Hradec Králové; \\ (Head: prof. MUDr. Z. Zadák, CSc.) \\ ** Department of Clinical Biochemistry \& Diagnostics, Faculty Hospital, Charles University, Hradec Králové;
}

(Head: doc. MUDr. V. Palička, CSc.)

\begin{abstract}
Summary: The detection of organic acidurias is a part of our screening programme for inherited metabolic diseases. Adapted procedure is differentiated and involves several steps: 1) thin-layer chromatography (TLC) in the case of an abnormal finding followed by 2) gas chromatography (GC). The next step of the investigation, using 3) gas chromatography mass-spectrometry (GS-MS) is reserved for more complicated and dubious analyses. In acutely sick patients and in the case of discrepancies between TLC results on the one hand, and clinical symptoms, supported by other laboratory findings on the other, the GC or GC-MS-analysis is performed immediately. Some examples of metabolic disorders, identified by this procedure, are presented.
\end{abstract}

Key words: Organic acids; Screening; Inherited metabolic disorders

\section{Introduction}

Profiling urinary organic acids is considered an invaluable tool for diagnosing the numerous metabolic diseases known as organic acidurias $(2-5,8)$. These are a group of genetic disorders in which an enzyme or cofactor defect in one of the metabolic pathways leads to accumulation and increased urinary excretion of specific acidic metabolites. Urinary organic acids (carboxylic acids and their amino acid conjugates) are water-soluble end products or intermediates of the metabolism of amino acids, sugars, lipids, steroids, biogenic amines and many other compounds. It should be pointed out that the products themselves are normal intermediates of the pathways involved, and it is the abnormal amounts present which is pathologic. Accumulated organic acids may be found in blood (organic acidemias), but in much lower concentration compared to urine (organic acidurias).

Organic acidurias manifest with an acute onset either early after birth or whenever later in life, often after provocation with a banal respiratory infect, vaccination or starvation. Otherwise, progression of neurological symptoms may be the only sign of disease.

Symptoms of the organic acidurias include vomiting, dehydration, tachypnoe, abnormal odour, seizures, hypotonia, hypertonia, ataxia, hepatomegalia, lethargy and death. Chronic manifestations are failure-to-thrive and mental retardation. Biochemical abnormalities, such as acidosis, hy- poglycemia and hyperammonemia are due to increased production of ketoacids and to the accumulated organic acid intermediates, which then inhibit other pathways.

Prompt diagnosis of the specific enzyme abnormality and early treatment may prevent life threatening episodes and psychomotor retardation in some disorders.

The variety of clinical features and the similarity of symptoms for completely different disorders increase the importance of identifying excreted organic acids, direct assay for deficient enzyme usually being either time-consuming or not widely available.

Recently we have introduced a three-step procedure for detection of organic acid abnormalities, the first using TLC methodology, the second GC and the third GC-MS technique.

\section{Experimental}

\section{Materials}

Organic acid standards were supplied by Sigma-Aldrich and Serva, silylating agents (BSTFA:TMCS) were from Pierce, precoated micropulverised cellulose thin-layers (plastic sheets No. 5577) and all other chemicals were purchased from Merck.

A fasting plasma and fresh random or 24-hrs-urine samples should preferably be taken before the therapy is introduced. Cerebrospinal fluid is analysed in neurometabolic diseases and vitreous humour in post-mortem diagnoses. 


\section{Methods}

High-performance thin layer chromatography (HPTLC)

We have adopted and slightly modified the method of Lee and Thurmon (6): Plasma or liquor are deproteinized with six times the volume of ethanol, after centrifugation the supernatant is concentrated to $1 \mathrm{ml}$. A volume of urine, equivalent to $2.2 \mu \mathrm{mol}(250 \mu \mathrm{g})$ of creatinine is diluted with water (or concentrated at $30{ }^{\circ} \mathrm{C}$ in vacuum) to $1 \mathrm{ml}$. Whenever the nitroso- $\beta$-naphthol test for ketoacids in urine was positive, oxime derivatives of $\alpha$-ketoacids were prepared by incubating urine at $\mathrm{pH} 14$ (alkalised with $\mathrm{NaOH}, 8$ mol. $\mathrm{l}^{-1}$ ) with aqueous hydroxylamine- $\mathrm{HCl}\left(25 \mathrm{~g} \mathrm{.}^{-1}\right)$ at $60{ }^{\circ} \mathrm{C}$ for $30 \mathrm{~min}$. After cooling, a volume equivalent to $250 \mu \mathrm{g}$ creatinine was further processed. All samples, supplemented from the beginning with $60 \mu \mathrm{g}$ phenylbutyric acid as an internal standard, were acidified to $\mathrm{pH} 1$, saturated with $\mathrm{NaCl}$, and vortex mixed for 5 min with $6 \mathrm{ml}$ of ether-ethylacetate, 1:1. After 10 min the organic layer was removed, concentrated under nitrogen to the volume of $1 \mathrm{ml}^{*}$ and $20 \mu \mathrm{l}$ were applied on the thin layer of cellulose. The plate was then stepwise $(1 \mathrm{~cm}$ further with each development) developed in the solvent system n-propanol - ammonium hydroxide, 2 mol..$^{-1}$ (7:3), using horizontal DS Chambers for TLC (Chromdes, Poland), with intermediate drying after each run and stained with anilinexylose reagent $\left(1 \mathrm{~g}\right.$ xylose in $3 \mathrm{ml} \mathrm{H} \mathrm{H}_{2} \mathrm{O}+1 \mathrm{ml}$ aniline in 96 $\mathrm{ml}$ methanol), diluted with methanol (2:1) before use.

\section{Gas chromatography $(G C)$}

As organic acids have low volatility, thermal instability and high polarity, derivatization is necessary prior to GC separation. The samples prepared for TLC were further processed for GC analysis, see footnote*. After spiking with an external standard (tetracosane) and evaporation to dryness under nitrogen, organic acids were derivatized with 100 $\mu 1$ of BSTFA-TMCS $(99: 1)$ at $60{ }^{\circ} \mathrm{C}$ for $30 \mathrm{~min}$ in a water bath. Trimethylsilyl derivatives in a volume of $1 \mu$ (equivalent to $22 \mathrm{ng}$ of creatinine) were analysed using capillary HP Ultra 2 column, $25 \mathrm{~m}$ x $0.33 \mathrm{~mm}$ ID, the Hewlett-Packard Model 5890 SE II with the oven temperature programmed from 50 to $300{ }^{\circ} \mathrm{C}, 5{ }^{\circ} \mathrm{C} / \mathrm{min}$ and a flame ionisation detector (7) controlled by HP ChemStation and HP RetIndex.

Gas chromatography-mass spectroscopy (GC-MS)

When needed, the same sample as that used for GC is further analysed using the Varian 3300 gas chromatograph with a fused silica capillary column DB1701 (J\&W), $30 \mathrm{~m} \times 0.25 \mathrm{~mm}$ ID, $0.25 \mu \mathrm{m}$ coating, coupled to an Finnigan Magnum ion trap. The Lauber injector and transfer line temperature is kept at $250{ }^{\circ} \mathrm{C}$ and $260^{\circ} \mathrm{C}$, respectively, the oven temperature program is set from 60 to $300^{\circ} \mathrm{C}$ with $5^{\circ} \mathrm{C} / \mathrm{min}$ increase. The parameters of ion trap are as follows: mass range from 50 to $550 \mathrm{amu}$, ionisation mode - electron impact, scan speed $4 \mu$ scans with auto ion control. The peaks are identified by reference to a mass spectral library.

\section{Strategy of examination}

1) Samples from acutely sick newborns with pronounced pathological laboratory results are immediately processed by GC (or GC-MS).

2) In all other cases the three-step examination is performed.

\section{Results and discussion}

HPTLC represents a prompt, inexpensive screening technique, used as the first approach in diagnostics of inherited metabolic diseases. The authors describing the procedure (6) claim quite reliable identification of samples with negative findings and those clearly pathological, provided that the child was examined promptly after the first symptoms had appeared and that it continuously received a normal amount of nutrients. This simple first-step procedure can be used in any general hospital laboratory, whenever an organic acid disorder is suspected.

The cost of GC discourages its use in a screening mode, therefore it is reserved for more advanced cases. GC provides high-efficiency resolution of numerous organic acids that are present in normal human urines. In a number of hereditary defects, organic acidurias are characterised mainly by quantitative changes of the normal acid profile, giving satisfactory results with GC alone. However, unknown compounds in some samples of the pathological urine, when detected by non-specific GC detectors such as the flame ionisation detector, could only be identified by the use of GC/MS.

Occasionally, we are simultaneously using both TLC and GC-MS, comparing the results. So far, our experience with the TLC is very good and several pathological conditions were detected, as illustrated in Figures 1 and 2.

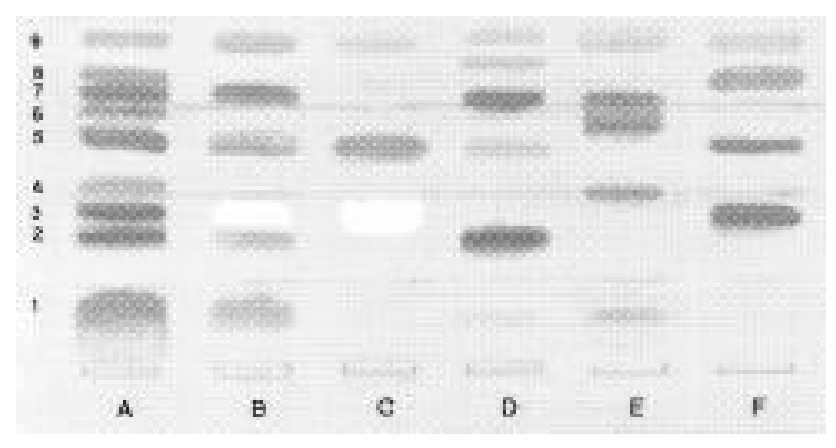

Figure 1. HPTLC of organic acids, multiple development, see text for details; A - standards: 1 - glutarate, 2 - methylmalonate, 3 - 3-hydroxy-3-methylglutarate, 4 - ascorbate, 5 - lactate, 6 - homogentisate, 7 - hippurate, 8 - 3-isovalerate, 9 phenylbutyrate; B - normal newborn, C - lactic aciduria, D methylmalonic aciduria, E - alcaptonuria, F - 3-hydroxy-3methylglutaric aciduria (B-F - urine samples).

\footnotetext{
* The same sample is further processed for gas chromatography, alternatively completed with mass spectrometry, if identification of abnormal or elevated organic acids is required.
} 


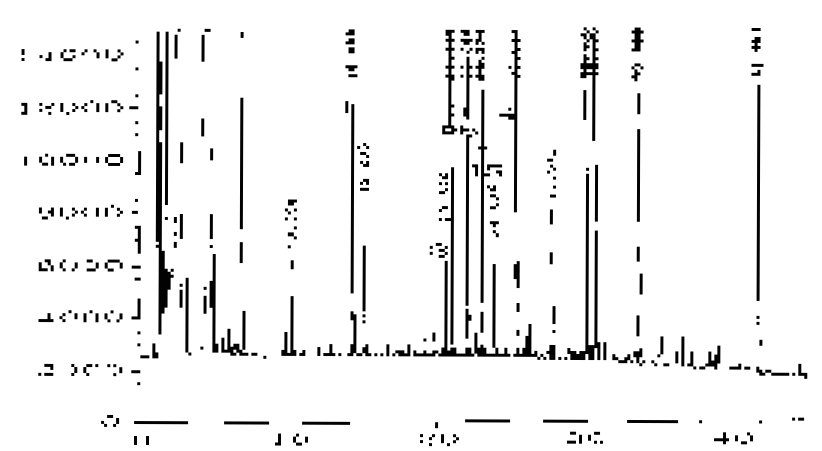

Figure 2. GC of urinary organic acids (trimethylsilyl derivatives) in a patient with 3-hydroxy-3-methylglutaryl-CoA lyase deficiency. 1 - 3-hydroxyisovaleric acid, 2 - 3-methylglutaric acid, 3 a,b - 3-methylglutaconic acid, cis, trans, 4 - 3-hydroxy-3-methylglutaric acid, IS - internal standard (phenylbutyric acid).

In general, analysis of organic acids is most reliable when the urine is collected during acute metabolic decompensation. Alternatively, the presence of an abnormality, which is associated with organic aciduria only intermittently, may be visible by provocative testing (by loading with metabolic intermediates or during starvation).

The determination of urinary organic acids contributes to the understanding of metabolic processes and serves as an important tool in the investigation of human diseases. In addition to organic acidurias, organic acid analysis may be an aid in the diagnosis of defects in other groups of metabolites. Examples are the presence of glycerol and glycerol3-phosphate in patients with fructose-1,6-diphosphatase deficiency or with type I tyrosinaemia or type I glycogenosis. It may assist in the diagnosis not only inherited but also acquired disorders, e.g. catecholamines metabolites in neural tumours or oxalic, glyoxylic, glycolic and glyceric acids in the hyperoxaluric syndromes (1).

The advantage of the proposed three-step procedure is the possibility of eliminating the clearly normal samples from further, economically far exigent processing by GC or GC-MS at the beginning of an investigation.

\section{Acknowledgements}

This study was supported by grant from the Czech Medical Research Council IGA MZ No. 4097-3.

\section{References}

1. Duran M et al. Group tests for selective screening of inborn errors of metabolism. Eur J Pediatr 1994; 153 (Suppl 1): $\mathrm{S} 27-\mathrm{S} 32$.

2. Hoffmann $\mathrm{G}$ et al. Quantitative analysis of organic acids in biological samples: batch isolation followed by gas chromatographic-mass spectrometric analysis. Clin Chem 1989;35:587-95.

3. Chalmers RA and Lawson A.M. Organic Acids in Man: Analytical Chemistry, Biochemistry, and Diagnosis of Organic Acidurias, Chapman and Hall, London, 1982.

4. Lehotay DC and Clarke JTR. Organic acidurias and related abnormalities. CRC Crit Rev Clin Lab Sci 1995;32/4: 377-429.

5. Tanaka $\mathrm{K}$ et al. Gas chromatographic method for analysis of urinary organic acid. II. Description of the procedure and its application to diagnosis of patients with organic acidurias. Clin Chem 1982;26:1847-53.

6. Lee BY, Thurmon TF. Practical clinical screening for organic acid disorders. LC-GC 1992;10(11):884-7.

7. Tuchman $\mathrm{M}$ et al. Capillary gas chromatographic separation of urinary organic acids. Retention indices of 101 urinary acids on a $5 \%$ phenylmethyl silicone capillary column. J Chromatogr Sci 1984;22:198-202.

8. Sweetmann L. Organic acid analysis. In: Hommes FA, ed. Techniques in diagnostic human biochemical genetics. A laboratory manual. New York: Wiley-Liss, 1991:143-76.

Submitted April 1997.

Accepted May 1997.

MUDr. Eliška Marklová, CSc., Baarova 1374, 50005 Hradec Králové, Czech Republic. 